ORIGINAL ARTICLE

\title{
The Effects of High-fat Diet on Histological Changes of Kidneys in Rats
}

\author{
Hotimah Masdan Salim ${ }^{1 *}$, Lintan Farizqi Kurnia ${ }^{1,}$, Tri Wahyuni Bintarti ${ }^{2}$, Handayani ${ }^{3}$ \\ ${ }^{1}$ Department of Biomedical Science, Faculty of Medicine, Nahdlatul Ulama Surabaya, Indonesia \\ ${ }^{2}$ Department of Molecularbiology, Faculty of Medicine, Nahdlatul Ulama University of Surabaya, Indonesia \\ ${ }^{3}$ Department of Pharmacology Medicine, Faculty of Medicine, Nahdlatul Ulama University of Surabaya, Indonesia
}

\section{A R T I C L E IN F O}

\section{Article history:}

Received 29 October 2018

Received in revised form 5

November 2018

Accepted 8 November 2018

Available online 30 November

2018

\section{Keywords:}

High-fat diet,

Rattus novergicus,

Kidney.

*) Corresponding author:

dr.hotimah@unusa.ac.id

\begin{abstract}
A B S T RA C T
Introduction Hypercholesterolemia contributes as a risk factor for endothelial dysfunction in the kidneys and worsens the condition of patients with previous kidney damage. This study aimed to examine the effect of hypercholesterol diet in kidney structure.

Methods: This was an experimental study, a case control using eight-week-old Rattus novergicus, divided into control and treatment group. High-fat diet (HFD) was administrated orally to treatment group for 14 days. All data statistically analyzed with two-paired ANOVA and post hoc for comparison.

Results: Histological analysis using hematoxylin-eosin staining showed degeneration in nephrons, including glomerulosclerosis, segmental necrosis and tubular defects. The mononuclear cell infiltration was Increased in HFD group significantly $(\mathrm{P}<0.05)$.

Conclusion: We concluded that a high fat diet induced obesity and may lead to renal deformities as a result of histopathological changes such as dilatation, tubular defects, inflammation and connective tissue enlargement of the kidney.
\end{abstract}

\section{Introduction}

Dyslipidemia amongst most important risk factor in cardiovascular disease and morbidity. The cardiovascular disease is one of the highest causes of death in Western countries. including among the most important risk factors is dyslipidemia, which is related to age and cause of death in Western countries. ${ }^{1}$

Genetic predisposition to hypercholesterolemia involves changes in lipoprotein transport and metabolism that causes atherosclerosis. ${ }^{2}$ Hypercholesterolemia contributes as a risk factor for endothelial dysfunction in kidney and worsens the condition of patients with previous kidney damage. ${ }^{3}$ Patients with dyslipidemia show cardiovascular manifestations, from several studies focusing on the effects of hypercholesterolemia which causes the progression of previous kidney disease. ${ }^{4}$

Kidney becomes the first organ that will experience damage from age and degenerative diseases. Renal aging is characterized by loss of function and increased glomerulosclerosis, tubular atrophy and interstitial fibrosis. ${ }^{5}$ The effect of aging in kidney damage pathology is also associated with changes in cholesterol metabolism. Previous experimental studies has shown that hypercholesterolemia can reduce renal blood flow, glomerular filtration and ultrafiltration, as well as damaging tubular and glomerular excretion. 6,7

Hypercholesterolemia can build up and develop into atherosclerosis, thus narrowing of blood vessels, especially in the heart, brain, kidneys, and eyes. Moreover, $22 \%$ of cardiac output passes through the kidneys, making them prone to endothelial capillaries damages. The endothelial damage results in microalbuminuria. ${ }^{8}$ In Indonesia, in patients with hypercholesterolemia found $23 \%$ experienced damage to the kidneys. In the MICRO-HOPE study, damage to the kidneys causes twice the risk of increased cardiovascular 
disease morbidity and mortality. Hypercholesterolemia can occur due to an unhealthy lifestyle, ranging from an unbalanced diet to a lack of sports activities. Unbalanced diet includes consumption of foods high in fat and carbohydrates, low in fiber, smoking habitsd also drinking alcohol. ${ }^{9}$

Based on the explanation above, this study intended to determine the effect of high-fat diet in histopathological changes in kidney rats model.

\section{Methods}

\section{Animal model}

Eight-week-old Rattus novergicus males were obtained from animal laboratories (Universitas Airlangga, Surabaya). The animals were acclimatized at room temperature $\left(25-30{ }^{\circ} \mathrm{C}\right)$ for one week; and had access to food and water ad libitum under a 12-hour light/dark cycle. After 1 week of acclimation, healthy Rattus novergicus males weight \pm 200 -250 gram were taken and divided into control and treatment group. In addition, the models were administrated with high-fat diet from cow fat emulsion as an induction of hyperlipidemia $2 \mathrm{ml}$ via oral gavage for 2 weeks. All experiment has been approved by the animal ethics committee of Universitas Airlangga and procedures conformed to guidelines for animal experimentation of Universitas Airlangga.

\section{Histological analysis}

The kidneys from each animal obtained after euthanized at day 14 and fixed in $10 \%$ formalin solution, then processed by the paraffin technique. Sections of $5 \mu \mathrm{m}$ thickness were cut and stained by haematoxylin and eosin (H\&E) for histological examination. Representative photomicrographs were obtained with a Olympus V-TVO 5XC-3 (Olympus, Tokyo, Japan) and microscope coupled to a Olympus camera using a $40 \times$ magnification objective.

\section{Statistical analysis}

All results were expressed as mean \pm standard error of mean (SEM). Comparison of parameters between two groups was performed with unpaired Student's t-test. Comparisons of dose-respons curves were made by twofactor repeated measures ANOVA, followed by Tukey's post hoc test for comparison between groups. A value of $\mathrm{P}<0.05$ was considered significant. All data analyzed using IBM SPSS Statistic for Windows version 19.0 (IBM Corp., Armonk, N.Y., USA).

\section{Results}

We evaluated these renal specimens of control group by light microscopy on an H\&E stained section, the following glomerular features were seen: the overall cellularity of the glomerulus, the symmetry of the glomerulus and the thickness of the capillary walls (fig. 1A). Renal tubules (the long and winding neck) formed as the proximal tubule, the loop of Henle, the distal tubule and proximal tubule (fig. 1B).
However, the histology in kidney sections from HFD-fed rats are summarized as follows. Dilatation in glomerular capillaries and other blood vessels was detected (fig.2A). Mononuclear cell infiltration was seen in the renal cortices of the HFD-fed rats (fig.2B). Degeneration in nephrons, including glomerulosclerosis, segmental necrosis and tubular defects, was found (fig.2B). There were many necrotic interstitial cells in the kidneys of the HFD-fed rats (fig.2B).

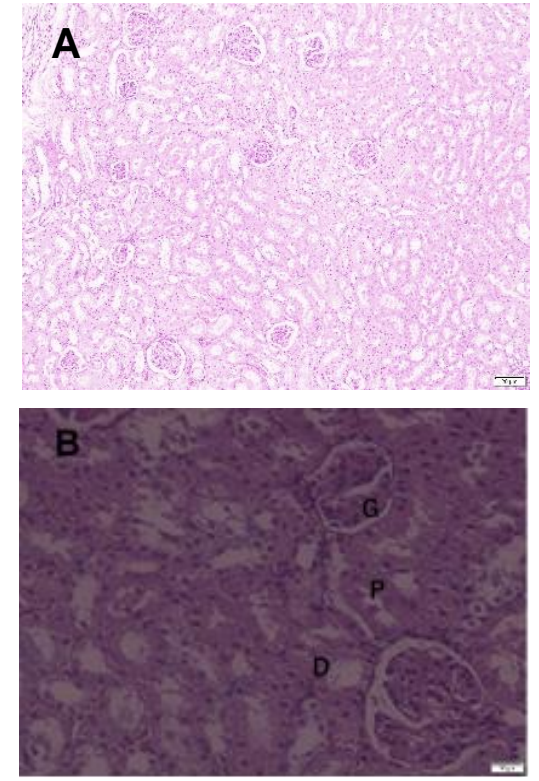

Figure 1. Light micrographs of kidney tissue from the control group. P, proximal tubule; D, distal tubule; G, glomerulus. Scale bars $=50 \mu \mathrm{m}$.

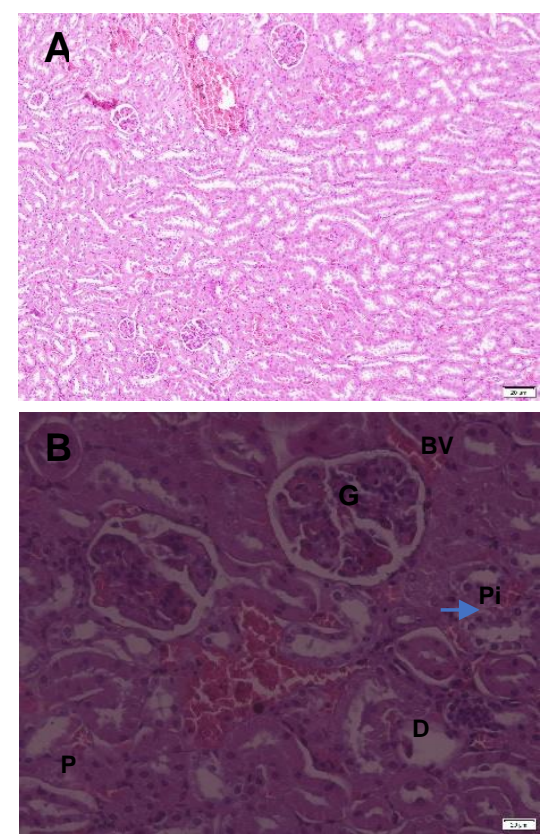

Figure 2. Light micrographs of kidney in the HFD group. $\mathrm{BV}$, dilated blood vessels; $\mathrm{P}$, proximal tubule; G, glomerulus. Inset reveals dilated glomerular capillaries (asterisk). Scale bars $=50 \mu \mathrm{m}$. 
On the basis of histological evidence of renal tissue inflammation in high-fat-diet induced obesity, the inflammation cells were examined. In high fat diet administration significantly increased the inflammation cells in histological kidney analysis compared with control group.

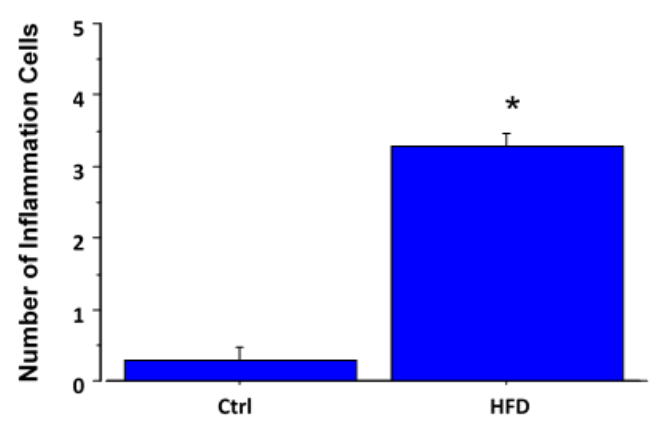

Figure 3. Increased renal inflammation in High-fat-diet induced obesity. High-fat-diet increased the inflammation cells in histological of kidney significantly compared with control group. All values are given as means \pm SEM. ${ }^{*} p<0.05$ control.

\section{Discussion}

Hypercholesterolemia is a general clinical problem that can cause complications in vital organ including kidneys. ${ }^{10}$ In previous studies with hypercholesterolemic experimental animals, hypercholesterolemia was found to interfere with lipid or cholesterol metabolism kidney. ${ }^{11}$ In previous studies of mice models given HFD found that disruption of lipid profile, kidney function and structure. ${ }^{11,12}$ In the present study, an obesity model was performed by feeding with animal tallow, and the effects of this obesity on rat kidneys were studied.

It is known that the proximal tubule is the first part of the kidney tubule which most substance functions are to filter and re-circulate blood to the system. Damaged in tubular can affect in nutrients and electrolytes loss. This study demonstrated that increases hypercholesterolemia affects the structure of the renal tubules. ${ }^{13}$ The changes are characterized by change in shape of the tubules and cells that are acidofilic. This study is in line with previous studies in which hypercholesterolemia induction causes tubular damage and inhibits filtration that causes protein loss and renal tubular luminal leak, ${ }^{14}$ and accumulation of fat droplets in tubular cells. ${ }^{15}$ The accumulation of fat droplets in tubular cells can affect cell metabolism and induce oxidative stress and lipid peroxidase. $^{16}$

In addition, research conducted stated that accumulation of fat in the renal tubules can produce lipotoxicity as fatty acids which act as detergents which can weaken the membrane structure and increase cell apoptosis.
The pervious study showed that excess lipids in the kidney lead to mitochondrial damage in all renal cell types, with reduced size, loss of cristae membranes, and decreased matrix density. Because the protein complexes of the electron transport chain are located on the cristae membranes, the loss of cristae membranes contributes to a loss of ATP production. The impact of mitochondrial dysfunction in the kidney appears to be cell-type specific. ${ }^{17}$ Mitochondrial dysfunction caused cell injury and death in glomerular endothelial cells, with $50 \%$ cell loss in HFD. ${ }^{18}$ However, in our study showed that HFD increased the inflammation cells in histological analysis of kidney significantly. The increasing of inflammation cells may related with lipid accumulation in the kidney. Furthermore, lipid accumulation is associated with reduced activity of AMPK in the kidney. ${ }^{19}$ The role of AMPK is to restore cellular energy balance by stimulating energy-generating pathways such as fatty acid oxidation and inhibiting fatty acid synthesis. Activation of AMPK with 5-aminoimidazole-4carboxamide-1-b-D-ribonucleoside prevented lipid accumulation and glomerulopathy in the kidney during HFD, but a direct role of AMPK in protecting the kidney is unclear because 5-aminoimidazole-4-carboxamide-1b-D-ribonucleoside also prevented weight gain, insulin resistance, and hyperglycemia, thus reducing metabolic stress to the kidney. ${ }^{20}$

\section{Conclusion}

In conclusion our study showed that a HFD induced obesity, and may lead to renal deformities as a result of histopathological changes such as dilatation, tubular defects, inflammation and connective tissue enlargement of the kidney.

\section{Conflict of Interest}

The author stated there is no conflict of interest.

\section{References}

1. Szadkowska I, Stanczyk A, Aronow WS, et al. Statin Therapy in the Elderly: A Review. Archives of Gerontology and Geriatrics. 2010; 50: 114-8.

2. Ross R. Atherosclerosis--An Inflammatory Disease. $N$ Engl J Med. 1999; 340: 115-26.

3. Bromfield $\mathrm{S}$ and Muntner P. High Blood Pressure: The Leading Global Burden of Disease Risk Factor and The Need for Worldwide Prevention Programs. Current Hypertension Reports. 2013; 15: 134-6.

4. Bruneval F. GW Approximation of the Many-Body Problem and Changes in the Particle Number. 2009, p.176403.

5. Percy CJ, Power D and Gobe GC. Renal Ageing: Changes in The Cellular Mechanism of Energy Metabolism and Oxidant Handling. Nephrology. 2008; 13: 147-52.

6. Tonkin AM. Clinical Relevance of Statins: Their Role in Secondary Prevention. Atherosclerosis Supplements. 2001; 2: 21-5.

7. Stulak JM, Lerman A, Porcel MR, et al. Renal Vascular Function in Hypercholesterolemia is Preserved by Chronic Antioxidant Supplementation. Journal of the American Society of Nephrology. 2001; 12: 1882-91.

8. Khonsary SA. Guyton and Hall: Textbook of Medical Physiology. Surgical Neurology International. 2017; 8: 275. 
9. OmniMed Communications. The MICRO-HOPE Study: Evidence for An Important Role for ACE Inhibitors. Diabetes \& Primary Care. 2018; 20: 9-10.

10. Nakhoul N and Batuman V. Role of Proximal Tubules in the Pathogenesis of Kidney Disease.

11. Otunola GA, Oloyede OB, Oladiji AT and Afolayan AA. Effects of Diet-induced Hypercholesterolemia on The Lipid Profile and Some Enzyme Activities in Female Wistar Rats. African Journal of Biochemistry Research. 2010; 4: 149-54.

12. Kwok C-y, Ngai-yan Wong C, Yin-Chun Yau M, et al. Consumption of Dried Fruit of Crataegus Pinnatifida (Hawthorn) Suppresses High-cholesterol Diet-induced Hypercholesterolemia in Rats. 2010.

13. Curthoys NP and Moe OW. Proximal Tubule Function and Response to Acidosis. Clinical Journal of the American Society of Nephrology : CJASN. 2014; 9: 1627-38.

14. Abdel-Hamid G. Effect of Red Grape Juice on Renal Glomeruli in Hypercholestremic Rats. 2014.

15. Zoja C, Abbate M and Remuzzi G. Progression of Renal Injury toward Interstitial Inflammation and Glomerular Sclerosis is Dependent on Abnormal Protein Filtration. Nephrology Dialysis Transplantation. 2015; 30: 706-12.

16. Balarini CM, Oliveira MZ, Pereira TM, et al. Hypercholesterolemia Promotes Early Renal Dysfunction in Apolipoprotein E-Deficient Mice. Lipids in Health and Disease. 2011; 10: 220-.

17. Szeto HH, Liu S, Soong Y, Alam N, Prusky GT and Seshan SV. Protection of Mitochondria Prevents High-fat Diet-induced Glomerulopathy and Proximal Tubular Injury. Kidney International. 2016; 90: 997-1011.

18. Amaral LS, Silva JA, Trindade TM, et al. Renal Changes in the Early Stages of Diet-induced Obesity in Ovariectomized Rats. Physiological Research. 2014; 63: 723-32.

19. Declèves A-E, Mathew AV, Cunard R and Sharma K. AMPK Mediates the Initiation of Kidney Disease Induced by A High-Fat Diet. Journal of the American Society of Nephrology : JASN. 2011; 22: $1846-55$

20. Declèves A-E, Zolkipli Z, Satriano J, et al. Regulation of Lipid Accumulation by AMK-activated Kinase in High Fat Diet Induced Kidney Injury. Kidney International. 2014; 85: 611-23. 